\title{
Investigation of Neutrophil/Lymphocyte Ratio and Mean Platelet Volume in Patients Diagnosed with Preterm Labor
}

\author{
Yüksel Kurban ${ }^{1 *}$, Ibrahim Uyar², Yasemin Alan ${ }^{3}$, Zeliha Atak ${ }^{4}$ and Önder Aydemir ${ }^{5}$ \\ ${ }^{1}$ Department of Obstetrics and Gynecology Clinic, Hight Specialized University Medical park Ankara Hospital, Turkey \\ ${ }^{2}$ Department of Obstetrics and Gynecology Clinic, Tepecik Training and Research Hospital, Turkey \\ ${ }^{3}$ Department of Obstetrics and Gynecology Clinic, Metropolitan Municipality Eşrefpaşa Hospital, Turkey \\ ${ }^{4}$ Department of Obstetrics and Gynecology Clinic, Keçiören Training and Research Hospital, Turkey \\ ${ }^{5}$ Public Health Center, Konya Provincial Directorate of Health, Turkey
}

Submission: July 07, 2020; Published: July 13, 2020

*Corresponding author: Yüksel Kurban, Department of Obstetrics and Gynecology, Hight Specialized University Medical park Hospital, Turkey

\begin{abstract}
Abstarct
Objective: Investigate the determining role of neutrophil/lymphocyte ratio (NLO) and mean platelet volume (MPV) in preterm birth in patients who gave birth before 37 weeks.

Method: A total of 138 patients were included in the study. Patients were divided into three groups: Early Preterm (delivery before 34 weeks, Group I=39), Late Preterm (delivery between 34 and 37 weeks, Group II=59) and the Control Group (delivery after 37 weeks, Group III=40). All three groups were compared in terms of demographic, obstetric and laboratory results, MPV and NLO parameters.

Results: There was no significant difference between the groups when the patients were compared in terms of age, gravida, parity, fetal sex and smoking $(\mathrm{p}>0.05)$. When the three groups were compared in terms of leukocyte, neutrophil, lymphocyte, hemoglobin, MPV and NLO, NLO was higher and MPV rate was lower in the preterm birth group, and the difference was significant $(\mathrm{p}<0.05)$. When the preterm delivery group was further divided as early preterm ( $<34$ weeks) and late (34-37 weeks) preterm delivery group, the NLO rate was higher in the former group, while MPV was lower and the difference was statistically significant $(\mathrm{p}<0.05)$.

Conclusion: NLO and MPV may be decisive as a proinflammatory process marker in patients who give birth before 37 weeks. Preterm births and fetuses of pregnant women with high NLO and low MPV may be considered to be likely to go to the neonatal care unit. men and the higher the family income, the more accurate oral health knowledge they have and the more correct oral Health care behavior.
\end{abstract}

Keywords: Neutrophil/Lymphocyte ratio; Mean Platelet Volume; Preterm labor

Abbreviation: AUC: Areaundercurve; CI: Confidentialinterval

\section{Introduction}

Preterm labor comprises $5-12 \%$ of all births and is still the main factor associated with perinatal morbidity and mortality worldwide [1]. Therefore, predicting preterm birth both prevents unnecessary interventions, especially in pregnancies before 32 weeks, and significantly reduces morbidity and mortality [2]. Intrauterine infection leading to delivery can be measured using various laboratory parameters. Systemic response to intrauterine infection is regulated by the natural immune system, which begins with the release of leukocyte types such as neutrophils and lymphocytes. An increase in the number of white blood cells or neutrophils is a sign of subclinical inflammation. Since inflammation and infection have important mechanisms in the etiology of preterm birth, there is a greater need to use a non-invasive method rather than invasive methods such as amniocentesis. In spontaneous preterm birth, neutrophil counts increase, and lymphocyte counts decrease, resulting in an increased NLO $[3,4]$. Women with a high neutrophil/lymphocyte 
ratio may be more likely to give preterm birth as they experience preterm birth symptoms. Cytokines released from the inflamed area in choriodecidua during the early stage of inflammation may result in a change in the number of leukocyte subsets [5]. On the other hand, in high-grade inflammatory conditions, the consumption of large platelets at the site of inflammation causes a decrease in MPV levels. It is well known that pregnancy itself and labor cause platelet activation. Therefore, low MPV levels are explained by the result of possible high-grade inflammation in preterm labor etiology $[6,7]$. In this study, we aimed to compare NLO and MPV ratios, which are inflammatory parameters that can predict the probability of preterm delivery in patients with preterm labor, among the patients with early preterm birth, late preterm birth and term delivery.

\section{Method}

This study was conducted by a retrospective examination of the patients who gave birth with preterm labor diagnosis from January 2017 to May 2018 at Ankara Keçiören Training and Research Hospital, Obstetrics and Gynecology Clinic. A total of 138 patients were included in the study. Patients were divided into three groups: Early Preterm (delivery before 34 weeks, Group I=39), Late Preterm (delivery between 34 and 37 weeks, Group $\mathrm{II}=59$ ) and the Control Group (delivery after 37 weeks, Group III=40). Patients with a history of metabolic disease, uterine anomalies, multiple pregnancies, fetal anomalies, intrauterine growth retardation, pregestational and gestational diabetes, amniotic membrane rupture, chorioamnionitis, fetal tachycardia, or patients with fever of unknown origin were excluded from the study. The control group consisted of healthy pregnant women who delivered between 37 and 42 weeks of gestation without any medical condition or poor obstetric features (diabetes, hypertension, obesity or earlier birth history according to gestational age). These three groups were compared in terms of demographic characteristics, obstetric and laboratory findings, maternal age, gravida, parity, smoking status, infant gender, neonatal weight, neonatal $1^{\text {st }}$ and $5^{\text {th }}$ minute Apgar scores, neonatal intensive care status, steroid administration, mode of delivery and levels of serum markers (leukocyte subtypes, platelet, leukocyte/ lymphocyte ratio (LLR) and mean platelet volume and hemoglobin levels. The first hemogram parameters were taken into account, which were measured when the patients were first admitted before delivery.

SPSS (Statistical Package for Social Sciences) for Windows 22.0 (SPSS Inc, Chicago, IL) was used for statistical analysis of the data obtained. Descriptive statistics were presented as mean \pm standard deviation (minimum-maximum), frequency distribution and percentage. Categorical variables were evaluated with Pearson's Chi-Square test. The compatibility of variables with normal distribution was examined using visual (histogram and probability graphs) and analytical methods (Kolmogorov Smirnov test). For the variables with normal distribution, Student's T-Test was used for the evaluation of statistical significance between the two independent groups, and for the variables that did not present a normal distribution, Mann-Whitney U-Test was used as the statistical method. The relationship between the variables was evaluated by Spearman's Correlation Test. The threshold of statistical significance was set at $\mathrm{p}<0.05$.

\section{Findings}

A total of 138 patients were included in the study, which consisted of 39 patients who had given birth before 34 weeks of gestation, 59 patients between 34 and 37 weeks, and 40 patients over 37 weeks. There was no statistical difference between these three groups in terms of age, parity, gravida, birth weight and smoking status $(\mathrm{P}>0.05)$ (Table 1$)$. The $\mathrm{C}$-section rate was $23 \%$ in Group I, 27\% in Group II and 7\% in Group III (Control Group). No significant difference was found between Groups I and II in terms of cesarean delivery compared with the control group $(\mathrm{p}<0.001)$ (Table 1).

Table 1: Distribution of some descriptive and clinical features among study groups.

\begin{tabular}{|c|c|c|c|c|c|}
\hline \multirow{2}{*}{$<34$ weeks $(n=39)$} & \multirow{2}{*}{$\begin{array}{c}\text { Total }(n=138) \\
\text { 34-37 weeks }(n=59)\end{array}$} & \multicolumn{3}{|c|}{ Gestatıonal Week } & \multirow[t]{2}{*}{$\mathbf{p}$} \\
\hline & & $\begin{array}{c}>37 \text { weeks }(n= \\
40)\end{array}$ & & & \\
\hline Age (year), mean $\pm S D(m i n-m a x)$ & $28.0 \pm 6.1(16-42)$ & $29.5 \pm 7.1(18-42)$ & $27.5 \pm 6.4(16-38)$ & $27.2 \pm 4.0(19-36)$ & $0.180 \mathrm{a}$ \\
\hline Gravida, mean \pm SD (min-max) & $2.3 \pm 1.3(1-6)$ & $2.4 \pm 1.2(1-6)$ & $2.5 \pm 1.4(1-6)$ & $2.0 \pm 1.0(1-5)$ & $0.251 \mathrm{~b}$ \\
\hline Parity, mean $\pm S D$ (min-max) & $1.1 \pm 1.0(0-4)$ & $1.2 \pm 1.0(0-4)$ & $1.1 \pm 1.1(0-4)$ & $1.0 \pm 1.0(0-4)$ & $0.778 \mathrm{~b}$ \\
\hline Surviving, mean \pm SD (min-max) & $1.1 \pm 1.0(0-4)$ & $1.1 \pm 1.0(0-4)$ & $1.1 \pm 1.0(0-4)$ & $1.0 \pm 1.0(0-4)$ & $0.802 \mathrm{~b}$ \\
\hline Smoking, n (\%) & $47(34.1)$ & $14(35.9)$ & $22(37.3)$ & $11(27.5)$ & $0.577 \mathrm{c}$ \\
\hline Mode of Delivery, n (\%) & & & & & \\
\hline
\end{tabular}




\begin{tabular}{|c|c|c|c|c|c|}
\hline NSVD & 81 (58.7) & $16(41.0)$ & $32(54.2)$ & $33(82.5)$ & \multirow{2}{*}{$0.001 \mathrm{c}$} \\
\hline $\mathrm{C} / \mathrm{S}$ & $57(41.3)$ & $23(59.0)$ & $27(45.8)$ & $7(17.5)$ & \\
\hline \multicolumn{6}{|l|}{ Baby's Gender, n (\%) } \\
\hline Female & $69(50.4)$ & $22(57.9)$ & $29(49.2)$ & $18(45.0)$ & \multirow{2}{*}{$0.507 \mathrm{c}$} \\
\hline Male & $68(49.6)$ & $16(42.1)$ & $30(50.8)$ & $22(55.0)$ & \\
\hline $\begin{array}{l}\text { Birth Weight }(g), \text { mean } \pm \text { SD } \\
\text { (min-max) }\end{array}$ & $\begin{array}{c}2523.1 \pm 807.6 \\
(980-4800)\end{array}$ & $\begin{array}{l}1660.0 \pm 411.5 \\
(980-2400) y z\end{array}$ & $\begin{array}{l}2422.3 \pm 361.3 \\
(1540-3195) \mathrm{z}\end{array}$ & $\begin{array}{c}3513.4 \pm 407.0 \\
(2850-4800)\end{array}$ & $<0.001 \mathrm{a}$ \\
\hline Steroid Administration, $\mathrm{n}(\%)$ & $39(28.3)$ & $25(64.1)$ & $14(23.7)$ & 0 & $<0.001 c$ \\
\hline PPROM, n (\%) & $25(18.1)$ & $9(23.1)$ & $15(25.4)$ & $1(2.5)$ & $0.009 c$ \\
\hline NIC Status, n(\%) & $78(56.5)$ & $38(97.4)$ & $33(55.9)$ & 7 (17.5) & $<0.001 \mathrm{c}$ \\
\hline
\end{tabular}

There were significant differences between the groups in terms of WBC levels, neutrophil levels, lymphocyte count and hemoglobin levels when compared regarding hemogram parameters $(\mathrm{p}<0.05)$ (Table 2$)$. MPV values were $7.5 \pm 1.8,9.0 \pm 2.5$ and $9.0 \pm 2.2$ respectively in groups I, II and III; NLO values were $6.1 \pm 3.0,4.5 \pm 2.5$ and $3.9 \pm 1.9$, respectively. When the three groups were compared in terms of MPV and NLO, the difference between them was significant. $(\mathrm{p}<0.05)$ (Table 2$)$.

Table 2: Distribution of some laboratory parameters between study groups.

\begin{tabular}{|c|c|c|c|c|c|}
\hline & \multirow{2}{*}{ Total $(n=138)$} & \multicolumn{3}{|c|}{ Gestatıonal Week } & \multirow{3}{*}{$\mathbf{p}$} \\
\hline & & $<34$ weeks $(n=39)$ & 34-37 weeks $(n=59)$ & $>37$ weeks $(n=40)$ & \\
\hline & $\begin{array}{l}\text { mean } \pm S D(\min - \\
\max )\end{array}$ & mean \pm SD (min-max) & mean $\pm S D(\min -\max )$ & mean $\pm S D(\min -\max )$ & \\
\hline WBC & $12.4 \pm 3.5(5.0-23.2)$ & $14.0 \pm 3.3(7.2-20.8) y z$ & $12.4 \pm 3.6(7.7-23.2)$ & $10.8 \pm 2.9(5.0-19.0)$ & $<0.001 \mathrm{a}$ \\
\hline Neutrophil & $9.1 \pm 3.2(3.6-19.4)$ & $10.0 \pm 3.2(5.6-18.0) \mathrm{z}$ & $9.3 \pm 3.4(4.5-19.4)$ & $7.9 \pm 2.5(3.6-15.7)$ & $0.015 \mathrm{a}$ \\
\hline Lymphocyte & $2.2 \pm 0.9(0.9-5.5)$ & $2.0 \pm 1.0(0.9-5.5) \mathrm{y}$ & $2.4 \pm 0.9(0.9-5.5)$ & $2.2 \pm 0.6(1.1-3.6)$ & $0.014 \mathrm{a}$ \\
\hline Hemoglobin & $11.8 \pm 1.4(7.1-14.7)$ & $11.3 \pm 1.2(7.1-13.7) \mathrm{z}$ & $11.9 \pm 1.6(7.3-14.7)$ & $12.1 \pm 1.2(9.3-14.0)$ & $0.036 \mathrm{~b}$ \\
\hline MPV & $8.6 \pm 2.3(4.7-17.7)$ & $7.5 \pm 1.8(4.7-11.9) \mathrm{yz}$ & $9.0 \pm 2.5(6.5-17.7)$ & $9.0 \pm 2.2(6.4-16.0)$ & $0.005 \mathrm{a}$ \\
\hline NLO & $4.8 \pm 2.6(1.3-14.3)$ & $6.1 \pm 3.0(1.8-13.8) y z$ & $4.5 \pm 2.5(1.3-14.3)$ & $3.9 \pm 1.9(2.0-11.6)$ & $0.001 \mathrm{a}$ \\
\hline \multicolumn{6}{|c|}{$\begin{array}{l}\text { n: Number of pregnant women; \%: Column percentage; mean: Mean; SD: standard deviation; a: Kruskal-Wallis test; b: One Way Analysis of Variance } \\
\text { (ANOVA); c: Pearson's Chi-Square Test; y: post-hoc bilateral comparison revealed a significant difference with "34-37 weeks"; z: Post-hoc bilateral } \\
\text { comparison revealed a significant difference with " }>37 \text { weeks" }\end{array}$} \\
\hline
\end{tabular}

A comparison of Group I and Group II in terms of MPV and NLO showed that MPV was lower in the early preterm group while NLO was higher, and the two groups presented a significant difference in terms of NLO and MPV $(p<0.05)$. A ROC curve analysis to determine the diagnostic quality of NLO and MPV to predict pregnancy under 34 weeks revealed that NLO $>4.73$ had $66 \%$ sensitivity and $64.4 \%$ specificity, whereas MPV $<7.96$ had 56.4 sensitivity and $57.6 \%$ specificity (Figure 1\&2) (Table 3). 


\section{Journal of Gynecology and Women's Health}

Table 3: Diagnostic sensitivity and specificity of serum markers in study groups.

\begin{tabular}{|c|c|c|c|c|c|}
\hline & Cutoff & Sensitivity (\%) & Specificity (\%) & PPD (\%) & NPD (\%) \\
\hline \multirow{2}{*}{ NLO } & 4.73 & 66.7 & 64.4 & 55.3 & 74.5 \\
\hline & 4.86 & 64.1 & 66.1 & 55.6 & 73.6 \\
\hline MPV & 7.96 & 56.4 & 57.6 & 77.3 & 71.1 \\
\hline
\end{tabular}

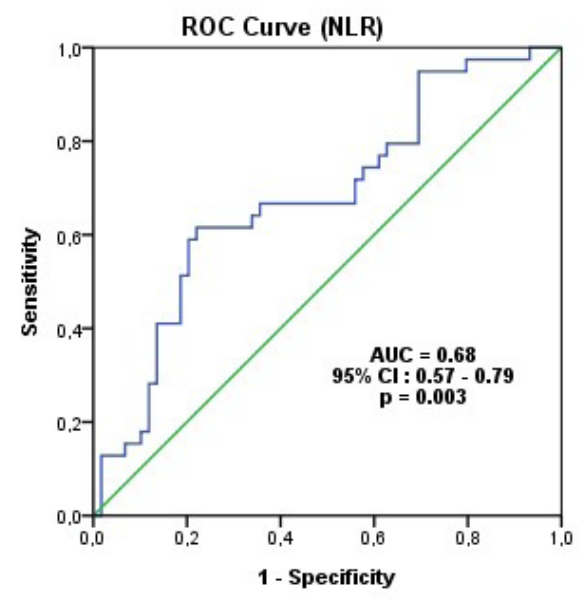

Figure 1: Receiver operating characteristic (ROC) curve for NLR values.

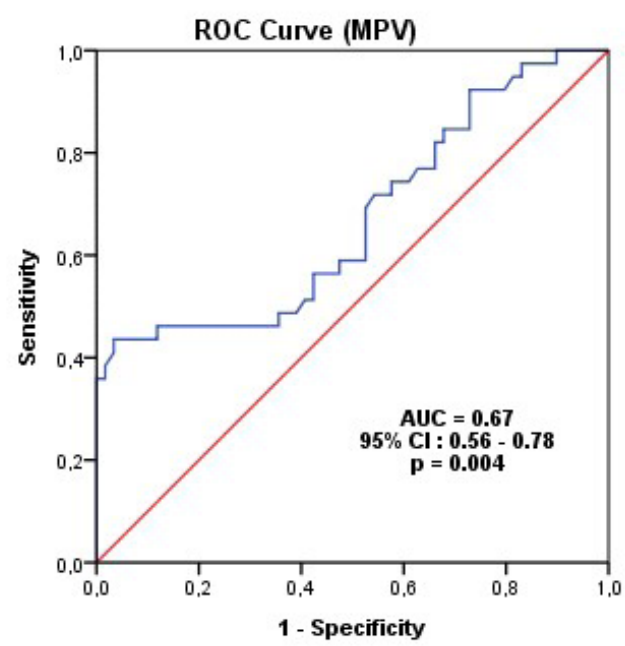

Figure 2: ROC curve for MPV values.

\section{Discussion}

The week of delivery has numerous effects on neonatal health in the short and long term. Preterm birth includes births before 37 weeks of gestation and accounts for approximately $5-12 \%$ of all births. ${ }^{1}$ Preterm birth is associated with high rates of neonatal mortality and morbidity, especially in long-term sequels, particularly before the week 32 [2]. About $28 \%$ of all neonatal deaths occurring in the first week of life can be associated with preterm birth [8]. Therefore, it is crucially important to predict and prevent preterm birth. As part of this study, we investigated 
the decisive role of NLO and MPV in patients diagnosed with preterm labor. In this study, the NLO ratio was higher in preterm groups compared with the control group while the MPV ratio was lower in the same group, which was consistent with the literature.

Preterm birth is a syndrome of multiple etiology, which can be induced by a series of causes including intrauterine infection, cervical disease, excessive tension on the uterus, uteroplacental ischemia or hemorrhage, endocrine disorders, stress and other immunologically mediated processes [9]. Many studies have proven that systemic and subclinical infections have a central role in preterm birth. Recent reports point to pathological inflammation in most preterm births $[10,11]$. It has been supported by many studies that immunity plays an active role in preterm labor and that infection is an important factor in early preterms. Multiple studies indicated that increased production of proinflammatory cytokines was associated with uterine activation and preterm birth, however, the production of anti inflammatory cytokines played an important role in the silence of the uterus during pregnancy $[12,13]$. Since the physiological immune response to various stressful events is often characterized by increased circulating neutrophils and decreased lymphocyte counts, some authors have suggested using NLO as an additional infection marker in clinical practice [14]. The mother's immune system plays an important role in all stages of human pregnancy; moreover, increased immune cells actively contribute to the initiation of the birth process by producing proinflammatory cytokines that cause uterine contraction. The labor is considered to be the last step of a proinflammatory cascade coordinated by an intrauterine environment linked to hormonal signals. In short, the inflammatory process plays a fundamental role in the pathogenesis of preterm birth [11]. Our study also supported this finding.

As the physiological immune response of circulating leucocytes to systemic inflammation accompanies increased neutrophils and decreased lymphocytes, NLO has been suggested as a simple systemic inflammation and stress parameter in various diseases $[3,4]$. Neutrophils are the most common type of leukocytes in the peripheral blood. Equipped with various antimicrobial effector mechanisms, neutrophils represent the first step of innate immune defense against most bacterial agents and are therefore considered to be the primary factors of acute inflammation. ${ }^{5}$ Many studies have shown the combination of NLO and cervical length to provide a more precise parameter than cervical length alone in predicting preterm birth [15]. Given that histological chorioamnionitis (HCA) is associated with increased blood inflammatory markers, it is certain that NLO is affected in histological HCA inflammatory processes. Increased NLO has been presented to be an appropriate indicator for the prenatal diagnosis of HCA [16]. Maternal NLO at birth offers more diagnostic benefits than CRP level in relation to acute HCA, and it may function as a useful marker in separating HCA without umbilical cord infection.

Immunity is a critical element of host defense against microbial invasion. In general, lymphopenia reflects the weakness of cellular immunity, while neutrophilia is a parameter indicating the response to systemic inflammation. In many studies, the ratio of these two values to each other is interpreted as indicating the adequacy of the cellular immune response against this condition despite the size of systemic inflammation $[3,5,14]$. Therefore, NLO can be interpreted as indicating the adequacy of the cellular immune response against this condition despite the extent of systemic inflammation. That is, in short, NLO is an indirect indicator of the host's immune response [10]. In addition, NLO is an affordable parameter that can be used to distinguish critical patients as an indicator of conveniently measurable and reproducible subclinical inflammation. In our study, NLO was increased in the preterm group and was found to be compatible with the literature.

Platelet indices such as platelet count, MPV, and PDW are routinely controlled whole blood (CBC) parameters. MPV is the measurement of platelet volume, which is among the parameters of this whole blood count. It is known to be an indicator of platelet function and activation. Given the latest data in the literature, it is claimed that platelets are not only involved in hemostasis, but also in the regulation of inflammatory responses occurring in the body. Tissue damage and subsequent inflammatory cytokine production lead to increased platelet production and activation. It is emphasized that this increase in platelet production also leads to a decrease in MPV value [17]. Natural growth factor sources including insulin-like growth factor 1 (IGF-1), plateletinduced growth factor (PDGF), vascular endothelial growth factor (VEGF) or growth factor $\beta$ (TGF- $\beta$ ) growth factor play important roles in different processes such as inflammation, angiogenesis, tissue repair regeneration [18]. Recent studies highlight the role of platelets and platelet-derived agents in thrombosis, angiogenesis, inflammation, and immunity [19]. Platelet activation occurs primarily in the process of hemostasis if the blood vessel is damaged. It can also occur in the process of acute and chronic inflammatory response. In preterm birth, some inflammatory cytokines are known to increase in both maternalfetal interface and systemic circulation [20]. Platelet activation indices such as MPV and PDW have been observed to vary more than controls in patients diagnosed with preterm labor due to inflammation and the likely ongoing ischemia process [13]. Some studies have demonstrated that the size and volume of platelets change differently in inflammation through certain cytokines: Although it has been shown that MPV levels could increase in low-grade inflammatory disorders with the participation of large platelets within the thrombus, the consumption of large platelets in the inflammatory site causes a decrease in the MPV levels in high grade inflammatory conditions [21,22]. It is well known that pregnancy itself and labor cause platelet activation [6,7]. The low MPV levels in our study were linked to likely high-grade inflammation in preterm labor etiology, and our findings were consistent with the literature. However, there are limited data on the relationship between platelet activation and preterm birth. A single study by Erez et al. [23] suggested that increased thrombin 
activation may cause platelet activation, which in turn induces preterm birth. We compared NLO and MPV levels with the control group over 37 weeks by grouping preterm birth patients as early preterm (before 34 weeks) and late preterm (34-37 weeks). In this study, NLO was increased in the preterm labor group, while MPV was lower in this group; and the findings were consistent with the literature. The limitation of our study was its retrospective design and that NLO and MPV were studied from the single blood sample taken. Therefore, we believe that further studies with larger samples are needed to determine whether the single blood sample taken accurately reflects NLO and MPV.

\section{Conclusion}

In conclusion, taking a full blood count (CBC) from all pregnant women is a routine. NLO and MPV are easily interpretable and promising hematologic parameters performed for each patient. They may be decisive as a proinflammatory process marker in patients who give birth before 34 weeks. Pregnant women with high NLO and low MPV may be considered to be likely to give preterm birth and their fetuses likely to go to the neonatal care unit. Measuring NLO and MPV in a pregnant woman experiencing preterm labor or preterm premature membrane rupture (PPROM) may reduce the frequency of unnecessary amniocentesis for chorioamnionitis as low as possible. Moreover, measuring NLO and MPV has other advantages, such as that the test sample can be obtained simply and easily measured using conventional facilities without the need for additional tools or reagents. As shown in this study and described in previous studies, maternal blood NLO and MPV can be considered a useful, non-invasive prenatal diagnosis method that is currently available. However, further studies are required to determine whether these parameters can be used to predict whether a pregnant woman at risk of preterm birth will result in adverse perinatal outcomes.

\section{What is Already Known on this Topic?}

Preterm birth is the leading cause of perinatal death and handicap in children and the vast majority of mortality and morbidity relates to early delivery before 37 week. The central role of systemic and subclinical infections in preterm labour is well documented. Intrauterine infection leading to delivery can be measured by using a variety of laboratory parameters. Preterm labor is associated with decreased mean platelet volume and increased neutrophil to lymphocyte ratio and they are an inexpensive, easily interpretable and promising haematologic parameters that is widely available.

\section{What this Study Adds?}

This study explored the association of high neutrophil to lymphocyte ratio and decreased mean platelet volume with to be likely to go to neonatal intensive care unit. Neutrophil to lymphocyte ratio and MPV could be used in combination with existing markers to improve detection rates of preterm birth.
Concomitant use of markers could be more powerful than measuring any of the individual markers alone.

\section{Authorship Contributions}

Medical Practices: Yüksel Kurban, İbrahim Uyar

Concept: Yüksel Kurban, Ibrahim Uyar, Yasemin Alan

Design: Yüksel Kurban, Ibrahim Uyar, Zeliha Atak, Yasemin Alan

Data Collection or Processing: Yüksel Kurban, Ibrahim Uyar, Zeliha Atak

Analysis or Interpretation: Önder Aydemir

Literature Search: Yüksel Kurban, Ibrahim Uyar, Yasemin Alan Writing: Yüksel Kurban, İbrahim Uyar.

\section{References}

1. Krupa FG, Faltin D, Cecatti JG, Souza JP (2006) Predictors of preterm birth. Int J Gynecol Obstet 94(1): 5-11.

2. Kramer MS, Demissie K, Yang H, Platt RW, Sauvé R, et al. (2000) The contribution of mild and moderate preterm birth to infant mortality. JAMA 284(7): 843-849.

3. Walsh SR, Cook EJ, Goulder F, Justin TA, Keeling NJ, et al. (2005) Neutrophil-lymphocyte ratio as a prognostic factor in colorectal cancer. J Surg Oncol 91(3): 181-184.

4. Nunez J, Nunez E, Bodi V, Sanchis J, Minana G, et al. (2008) Usefulness of the neutrophil to lymphocyte ratio in predicting long-term mortality in ST segment elevation myocardial infarction. Am J Cardiol 101(6): 747-752.

5. Simcox R, Sin WT, Seed PT, Briley A, Shennan AH, et al. (2007) Prophylactic antibiotics for the prevention of preterm birth in women at risk: a meta- analysis. Aust N Z J Obstet Gynaecol 47(5): 368-377.

6. Dundar O, Yoruk P, Tutuncu L (2008) Longitudinal study of platelet size changes in gestation and predictive power of elevated MPV in development of preeclampsia. Prenat Diagn 28(11): 1052-1056.

7. Beyan C, Kaptan K, Ifran A (2006) Platelet count, mean platelet volume, platelet distribution width, and plateletcrit do not correlate with optical platelet aggegation responses in healthy volunteers. J Thromb Thrombolysis 22(3): 161-164.

8. Beck T, Wojdyla D, Say L (2010) The worldwide incidence of preterm birth: A systematic review of maternal mortality and morbidity. Bull World Health Organ 88(1): 31-38.

9. Slattery MM, Morrison JJ (2002) Preterm delivery. Lancet 360(9344): 1489-1497.

10. Riley K, Nelson DM (2010) Toll-like receptors in pregnancy disorders and placental dysfunction Clin. Rev Allergy Immunol 39(3): 185-193.

11. Kalagiri RR, Carder T, Choudhury S, Vora N, Ballard AR, et al. (2016) Inflammation in complicated pregnancy and its outcome. Am J Perinatol 33(4): 1337-1356.

12. Challis JR, Lockwood CJ, Myatt L, Norman JE, Strauss III, et al. (2009) Inflammation and pregnancy. Reprod Sci 16(2): 206-215.

13. Gasparyen AY, Ayvazyen L, Mikhalidis D (2011) MPV: A link between thrombosis and inflammation? Curr Pharm Des 17(1): 47-58. 
14. Alice Gilman-Sachs, Svetlana Dambaeva, Salazar Garcia MD, Youssef Hussein, Joanne Kwak-Kim, et al. (2018) Inflammation induced preterm labor and birth. J Reprod Immunol 129: 53-58.

15. Kim MA, Lee BS, Park YW, Seo K (2011) Serum markers for prediction of spontaneous preterm delivery in preterm labour. Eur J Clin Invest 41(7): 773-780.

16. Cappelletti M (2016) Inflammation and preterm birth. J Leukoc Biol 99(1): 67-78.

17. Incebiyik A, Seker A, Vural M, Hilali NG, Camuzcuoglu A, et al. (2014) May mean platelet volume levels be a predictor in the diagnosis of pelvic inflammatory disease? Wien Klin Wochenschr 126(13-14): 422426

18. Turgut A, Sak ME, Ozler A (2013) Alterations of peripheral blood cells in tubal ectopic pregnancy. Ginekol Pol 84(3): 193-196.
19. Wagner DD, Burger PC (2003) Platelets in inflammation and thrombosis. Arterioscler Thromb Vasc Biol 23(12): 2131-2137.

20. Turhan NO, Karabulut A, Adam B (2000) Maternal serum interleukin 6 levels in preterm labor: prediction of admission-to-delivery interval. J Perinat Med 28(2): 133-139.

21. Holmes VA, Wallace JM, Gilmore WS, McFaul P, Alexander HD, et al. (2002) Soluble P-selectin levels during normal pregnancy: a longitudinal study. BJOG 109(9): 997-1002.

22. Holthe MR, Staff AC, Berge LN, Lyberg T (2004) Different levels of platelet activation in preeclamptic, normotensive pregnant, and nonpregnant women. Am J Obstet Gynecol 190(4): 1128-1134.

23. Erez O, Romero R, Hoppensteadt D, Chaiworapongsa T, Kusanovic JP, et al. (2008) Premature labor: a state of platelet activation. J Perinat Med 36(5): 377-387.

\section{Your next submission with Juniper Publishers} will reach you the below assets

- Quality Editorial service

- Swift Peer Review

- Reprints availability

- E-prints Service

- Manuscript Podcast for convenient understanding

- Global attainment for your research

- Manuscript accessibility in different formats

( Pdf, E-pub, Full Tsext, Audio)

- Unceasing customer service

Track the below URL for one-step submission https://juniperpublishers.com/online-submission.php 